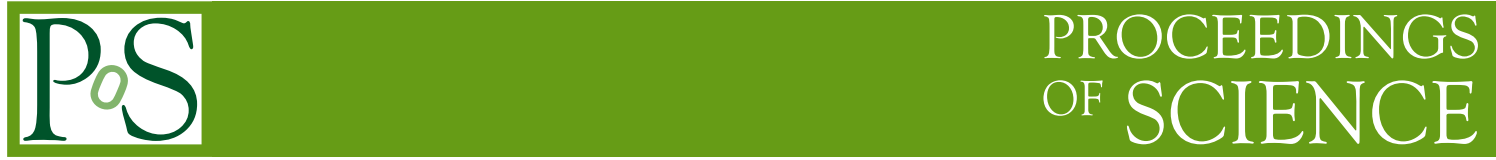

\title{
Non-linear QCD dynamics and exclusive production in $e p$ collisions
}

\author{
M.V.T. Machado* \\ High Energy Physics Phenomenology Group, GFPAE IF-UFRGS \\ Caixa Postal 15051, CEP 91501-970, Porto Alegre, RS, Brazil \\ E-mail: magnusdif.ufrgs.br
}

\section{V.P. Gonçalves and A.R. Meneses}

Instituto de Física e Matemática, Universidade Federal de Pelotas

Caixa Postal 354, CEP 96010-900, Pelotas, RS, Brazil.

In this contribution we analyse the cross sections for the exclusive vector meson production as well as the deeply virtual Compton scattering (DVCS) relying on the color dipole approach and considering the numerical solution of the Balitsky-Kovchegov equation including running coupling corrections. Comparisons to DESY-HERA data on exclusive processes and predictions to the Large Hadron Electron Collider, LHeC, are presented.

35th International Conference of High Energy Physics - ICHEP2010,

July 22-28, 2010

Paris France

\footnotetext{
* Speaker.
} 


\section{Introduction}

At high energies, the growth of the parton distribution is expected to saturate, forming a Color Glass Condensate (CGC), whose evolution with energy is described by an infinite hierarchy of coupled equations for the correlators of Wilson lines [1]. In the mean field approximation, the first equation of this hierarchy decouples and boils down to a single non-linear integro-differential equation: the Balitsky-Kovchegov (BK) equation [2]. This equation determines, in the large- $N_{c}$ (the number of colors) limit, the evolution of the two-point correlation function, which corresponds to the scattering amplitude $\mathscr{N}(x, r, b)$ of a dipole off the CGC, where $r$ is the dipole size and $b$ the impact parameter. This quantity encodes the information about the hadronic scattering and then about the non-linear and quantum effects in the hadron wave function. Recently, the next-to-leading order corrections to BK equation were calculated [3] through the ressumation of $\alpha_{s} N_{f}$ contributions to all orders, where $N_{f}$ is the number of flavors. Such calculation allows one to estimate the soft gluon emission and running coupling corrections to the evolution kernel and it was found that the dominant contributions come from the running coupling corrections, which allows to determine the scale of the running coupling in the kernel. More recently, a global analysis of the small $x$ data for the proton structure function using the improved BK equation was performed [4]. In contrast to the BK equation at leading logarithmic $\alpha_{s} \ln (1 / x)$ approximation, which fails to describe data, the inclusion of running coupling effects to evolution renders BK equation compatible with them.

Exclusive processes in deep inelastic scattering (DIS) have appeared as key reactions to trigger the generic mechanism of diffractive scattering. In particular, the diffractive vector meson production and deeply virtual Compton scattering (DVCS) have been extensively studied at HERA and provide a valuable probe of the QCD dynamics at high energies. In a general way, these processes are driven by the gluon content of target (proton or nuclei) which is strongly subject to parton saturation effects as well as considerable nuclear shadowing corrections when one considers scattering on nuclei. In particular, the cross section for exclusive processes in DIS are proportional to the square of scattering amplitude, which turn it strongly sensitive to the underlying QCD dynamics. In this contribution we summarize the main results published in Ref. [5] where we make use of numerical solution of the BK equation including running coupling corrections (BK RC) in order to estimate the contribution of the saturation physics for exclusive processes. Our analysis is relevant for the physics to be studied in future electron - proton collider, as e.g. the LHeC [6]. In next section we provide the main formula for computing the differential cross section for exclusive processes in DIS and discuss the numerical results considering the solution of the BK RC model.

\section{Exclusive processes in DIS and the RC BK solution}

Let us consider photon-hadron scattering in the dipole frame, in which most of the energy is carried by the hadron, while the photon has just enough energy to dissociate into a quark-antiquark pair before the scattering. In this representation the probing projectile fluctuates into a quarkantiquark pair (a dipole) with transverse separation $r$ long after the interaction, which then scatters off the hadron. In the dipole picture the amplitude for production of an exclusive final state $E$, such as a vector meson $(E=V)$ or a real photon in DVCS $(E=\gamma)$ is given by:

$$
\mathscr{A}_{T, L}^{\gamma^{*} p \rightarrow E p}\left(x, Q^{2}, \Delta\right)=i \int d z d^{2} r d^{2} b e^{-i[b-(1-z) r] \cdot \Delta}\left(\Psi_{E}^{*} \Psi\right)_{T} 2 \mathscr{N}(x, r, b)
$$



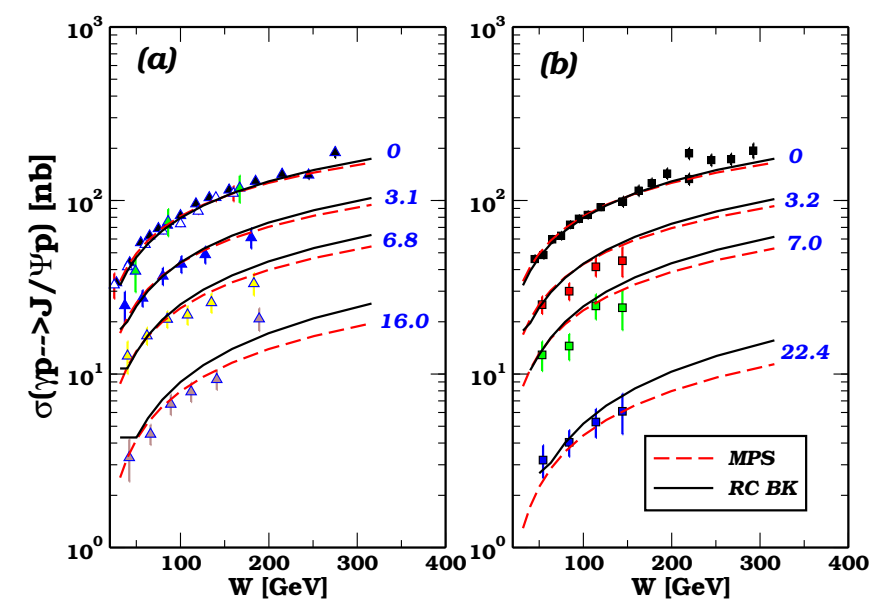

Figure 1: Energy dependence of the $\gamma p$ cross section for $J / \Psi$ production for different photon virtualities. Data from (a) ZEUS and (b) H1 collaborations (see Ref. [5] and references therein).

where the factor $[i(1-z) r] . \Delta$ in the exponential arises when one takes into account non-forward corrections to the wave functions. Therefore, the differential cross section for exclusive production is given by

$$
\frac{d \sigma_{T, L}}{d t}\left(\gamma^{*} p \rightarrow E p\right)=\frac{1}{16 \pi}\left|\mathscr{A}_{T, L}^{\gamma^{*} p \rightarrow E p}\left(x, Q^{2}, \Delta\right)\right|^{2}\left(1+\beta^{2}\right),
$$

where $\beta$ is the ratio of real to imaginary parts of the scattering amplitude. For the case of heavy mesons, skewness corrections are quite important and they are also taken into account. For the meson wavefunction, we have considered [5] the Gauss-LC model which is a simplification of the DGKP wavefunctions. In photoproduction, this leads only to an uncertainty of a few percents in overall normalization. We consider the quark masses $m_{u, d, s}=0.14 \mathrm{GeV}, m_{c}=1.4 \mathrm{GeV}$ and $m_{b}=4.5 \mathrm{GeV}$.

The scattering amplitude $\mathscr{N}(x, r, b)$ contains all information about the target and the strong interaction physics. In the Color Glass Condensate (CGC) formalism [1], it encodes all the information about the non-linear and quantum effects in the hadron wave function. In leading order (LO), and in the translational invariance approximation - in which the scattering amplitude does not depend on the collision impact parameter $b$ - it reads

$$
\begin{aligned}
\frac{\partial \mathscr{N}(r, Y)}{\partial Y} & =\int \mathrm{d} r_{1} K^{\mathrm{LO}}\left(r, r_{1}, r_{2}\right)\left[\mathscr{N}\left(r_{1}, Y\right)+\mathscr{N}\left(r_{2}, Y\right)-\mathscr{N}(r, Y)-\mathscr{N}\left(r_{1}, Y\right) \mathscr{N}\left(r_{2}, Y\right)\right], \\
K^{\mathrm{LO}}\left(r, r_{1}, r_{2}\right) & =\frac{N_{c} \alpha_{s}}{2 \pi^{2}} \frac{r^{2}}{r_{1}^{2} r_{2}^{2}},
\end{aligned}
$$

where $\mathscr{N}(r, Y)$ is the scattering amplitude for a dipole (a quark-antiquark pair) off a target, with transverse size $r, Y \equiv \ln \left(x_{0} / x\right)$ ( $x_{0}$ is the value of $x$ where the evolution starts), and $r_{2}=r-r_{1}$. This equation is a generalization of the linear BFKL equation (which corresponds of the first three terms), with the inclusion of the (non-linear) quadratic term, which damps the indefinite growth of the amplitude with energy predicted by BFKL evolution. From recent numerical studies of 
the improved BK equation, it has been confirmed that the running coupling corrections lead to a considerable increase in the anomalous dimension and to a slow-down of the evolution speed, which implies, for example, a slower growth of the saturation scale with energy, in contrast with the faster growth predicted by the LO BK equation. Moreover, as shown in [4] the improved BK equation has been shown to be really successful when applied to the description of the ep HERA data for the inclusive proton structure function.

In what follows we show some results of RC BK approach compared to the experimental data on exclusive processes at DESY-HERA and present our predictions. As a sample of studies presented in Ref. [5], Fig. 1 presents the predictions of the RC BK model for the diffractive $J / \Psi$ production and compare them to the ZEUS (a) and H1 (b) data [5]. The data description is reasonable since it is a parameter-free calculation. The contribution of real part of amplitude increase by $10 \%$ the overall normalization, while the skewedness have a $20 \%$ effect [5]. For comparison, we show also the results for the Marquet-Peschanski-Soyez model [7] (MPS) for the dipole cross section.

Let us now perform a preliminary study for the LHeC machine [6]. Using the design with a electron beam having laboratory energy of $E_{e}=140 \mathrm{GeV}$, the center of mass energy will reach $W_{\gamma p}=1.4 \mathrm{TeV}$ and a nominal luminosity of order $10^{33} \mathrm{~cm}^{-2} \mathrm{~s}^{-1}$. Our estimates for the exclusive vector meson photoproduction cross sections are the following. One gets $\sigma(\gamma+p \rightarrow V+p) \simeq 18$ $\mu \mathrm{b}, 503 \mathrm{nb}$ and $3.4 \mathrm{nb}$ for $\rho^{0}, \psi(1 S)$ and $\Upsilon(1 S)$, respectively. The average error is of order $10 \%$, which includes uncertainties on the meson wavefunctions, elastic slope and skewedness effects. For DVCS, we estimate the value $\sigma\left(\gamma^{*}+p \rightarrow \gamma+p\right) \simeq 28 \mathrm{nb}$ at photon virtuality of $Q^{2}=8 \mathrm{GeV}^{2}$, with a few percent error due to elastic slope and skwedness modeling.

As a summary, we presented an analysis of exclusive production in small- $x$ deep inelastic scattering in terms of the non-linear QCD dynamics. This approach was performed using the recent calculation of the running coupling corrections to the BK equation. We find a fairly good agreement with experimental data using a parameter-free calculation and provide predictions to the $\mathrm{LHeC}$ experiment. Our predictions are relevant for the physics programs in the ongoing experiment $\mathrm{LHeC}$ and in the photoproduction processes in coherent proton - proton interactions at the LHC.

\section{References}

[1] F. Gelis, E. Iancu, J. Jalilian-Marian and R. Venugopalan, Annual Review of Nuclear and Particle Sciences, vol. 60 (2010), arXiv:1002.0333 [hep-ph].

[2] I. I. Balitsky, Nucl. Phys. B463, 99 (1996); Y.V. Kovchegov, Phys. Rev. D 60, 034008 (1999); Phys. Rev. D 61074018 (2000).

[3] Y. V. Kovchegov and H. Weigert, Nucl. Phys. A 784, 188 (2007); J. L. Albacete and Y. V. Kovchegov, Phys. Rev. D 75, 125021 (2007); I. Balitsky, Phys. Rev. D 75, 014001 (2007); I. Balitsky and G. A. Chirilli, Phys. Rev. D 77, 014019 (2008).

[4] J. L. Albacete, N. Armesto, J. G. Milhano and C. A. Salgado, Phys. Rev. D80, 034031 (2009).

[5] V.P. Gonçalves, M.V.T. Machado and A.R. Meneses, Eur. Phys J C68, 133 (2010).

[6] J. B. Dainton, M. Klein, P. Newman, E. Perez and F. Willeke, JINST 1, P10001 (2006)

[7] C. Marquet, R. B. Peschanski and G. Soyez, Phys. Rev. D 76, 034011 (2007). 